\title{
Propuesta metodológica para el análisis territorial de las áreas empresariales. Especial referencia al norte de España
}

\author{
Methodological proposal for the territorial analysis \\ of business areas. Special reference to Northern Spain
}

\author{
Paz Benito del Pozo ${ }^{1}$ y César Luna Rabanal ${ }^{2}$
}

\section{INTRODUCCIÓN}

Este trabajo tiene su origen en un proyecto de investigación centrado en analizar los aspectos de ordenación, planificación y gestión de las áreas empresariales en España. El objetivo último es establecer el modelo territorial de la industria que se deriva de las políticas territorial e industrial que tienen su reflejo en los documentos y normas que regulan la producción de suelo para actividades económicas en el norte del país desde principios de la década de 1990 hasta la actualidad. Para ello se toman como referencia las Comunidades Autónomas de Galicia, Asturias, Castila y León, Cantabria, País Vasco y Navarra, y se acotan cuatro aspectos clave para comprender la organización espacial y la estructura de las áreas empresariales:

a) Las pautas que rigen la ordenación de los espacios para actividades económicas.

b) Los principios y criterios que sustentan la planificación oficial de suelo industrial.

\footnotetext{
${ }^{1}$ Departamento de Geografía y Geología de la Universidad de León: paz.benito@unileon.es

2 Departamento de Geografía y Geología de la Universidad de León: cluna.rabanal@gmail.com
} 
c) El desarrollo urbanístico de los asentamientos para empresas.

d) Los instrumentos de gestión y gobernanza que los agentes públicos y privados aplican a los asentamientos empresariales.

Para avanzar en el análisis de los aspectos indicados ha sido preciso ensayar una metodología que combina técnicas cualitativas y cuantitativas convencionales con el desarrollo de herramientas digitales originales y la ejecución de algunas operaciones de investigación tentativas con las que obtener respuestas satisfactorias a los problemas planteados. Así, la propia exploración del método se convierte en un fin en sí mismo, se somete a discusión en diversas convocatorias científicas y se depura hasta llegar a la propuesta que aquí se expone.

El propósito de este trabajo es explicar y poner a disposición de la comunidad científica una metodología rigurosa y con elementos de novedad aplicada a la identificación, toma de datos y tratamiento de la información sobre las áreas empresariales, lo que habrá de permitir una clasificación por tipologías homogéneas y un análisis territorial de los espacios productivos a diferentes escalas.

\section{ESCALAS A CONSIDERAR: AGENTES Y TERRITORIO}

La escala del análisis territorial de las áreas empresariales es modulable y se ajusta a la delimitación de las unidades administrativas en que se divide el territorio español (Comunidades Autónomas, provincias y municipios). Asimismo está condicionada por el ámbito de afectación de los documentos que rigen los aspectos de ordenación y planificación de los espacios industriales y empresariales: la región o Comunidad Autónoma para el estudio de los aspectos relativos a la ordenación de las actividades económicas (que se expresa en las Directrices Regionales de Ordenación del Territorio y Planes de Ordenación Subregionales) y también para el análisis de la planificación oficial de suelo industrial (Planes de Suelo Industrial promovidos por los Gobiernos autonómicos); la provincia para ciertas acciones de creación de suelo empresarial impulsadas por las Cámaras de Comercio e Industria o las Diputaciones; y el municipio para el estudio de los aspectos urbanísticos que rigen el desarrollo de los asentamientos empresariales, tanto en medio rural como urbano, y que son competencia de los Ayuntamientos (Planes Generales de Ordenación Urbana, Normas Urbanísticas, Planes Parciales, etcétera). 
Por su parte, la gestión y la gobernanza de las áreas empresariales implica a un conjunto heterogéneo de agentes privados (promotores de suelo, asociaciones de polígonos y parques, organizaciones empresariales) y públicos (agencias de desarrollo, corporaciones locales, entes estatales) y sus efectos se difunden por el territorio de forma vertical o multiescalar. Por ejemplo, la Coordinadora Española de Polígonos Empresariales (CEPE) y la Asociación de Parques Científicos y Tecnológicos de España (APTE) proyectan su acción gestora a escala nacional, atendiendo a los requerimientos de sus asociados, pero también promueven acciones específicas a escala regional o local en respuesta a las necesidades o demandas de territorios y socios concretos. La primera agrupa a federaciones y asociaciones de áreas empresariales o cualquier otra entidad que esté formada por empresarios o propietarios y vinculada a la gestión, funcionamiento y dinamización de las áreas empresariales, siendo su principal objetivo conseguir infraestructuras adecuadas y correctamente gestionadas, en las que los servicios se presten de acuerdo a las necesidades de las empresas, incluidos los servicios avanzados (ver http://www.poligonoscepe.org/; Verificada el 22/03/2015); la segunda, APTE, es una pieza importante del sistema español de Ciencia-TecnologíaEmpresa y sus miembros son parques científicos y tecnológicos repartidos por todo el país, es decir, 47 socios operativos que concentran a más de 6.000 empresas e instituciones (ver http://www.apte.org/es/; Verificada el 22/03/2015).

También es preciso contemplar el alcance y magnitud de la acción promotora de los agentes públicos que proyectan desarrollos de suelo para actividades empresariales. De nuevo se reproducen las escalas administrativas. Así, organismos como SEPES (Entidad Estatal de Suelo) y SEPI (Sociedad Estatal de Participaciones Industriales) construyen (en el pasado y en el presente) polígonos y parques empresariales en todo el territorio nacional, pues son instrumentos del Gobierno central y sus acciones expresan la política en materia de suelo para actividades económicas (entre otros cometidos). Por su parte, los Gobiernos autonómicos tienen sus propios instrumentos de creación de suelo industrial (IDEPA en Asturias; SODERCAN en Cantabria; SPRILUR en el País Vasco, ADE en Castilla y León, etcétera), a los que se suma la acción a escala provincial de las Cámaras de Comercio e Industria o de las Diputaciones; y a escala local de Ayuntamientos, Cámaras, Consorcios, Zonas Francas, etcétera. En suma, un complejo entramado institucional que se resume en el cuadro 1 para el ámbito territorial de este estudio. 
Cuadro 1

AGENTES PROMOTORES Y GESTORES DE ÁREASEMPRESARIALES EN EL NORTE DE ESPANA

\begin{tabular}{|c|c|c|c|c|}
\hline CC AA & Estatal & Regional & Provincial & Municipal/local \\
\hline Asturias & $\begin{array}{l}\text { - SEPES } \\
\text { - SEPIDES } \\
\text { - CEPE } \\
\text { - APTE }\end{array}$ & $\begin{array}{l}\text { - IDEPA } \\
\text { - SOGEPSA }\end{array}$ & & $\begin{array}{l}\text { - Ayuntamientos } \\
\text { - Cámaras de C. I. } \\
\text { de Oviedo, Gijón, } \\
\text { Avilés } \\
\text { - Valnalón }\end{array}$ \\
\hline Cantabria & $\begin{array}{l}\text { - SEPES } \\
\text { - SEPIDES } \\
\text { - CEPE } \\
\text { - APTE }\end{array}$ & $\begin{array}{l}\text { - SODERCAN } \\
\text { - SICAN }\end{array}$ & $\begin{array}{l}\text { - Cámara de } \\
\text { Comercio e } \\
\text { Industria } \\
\text { de Cantabria }\end{array}$ & $\begin{array}{l}\text { - Ayuntamientos } \\
\text { - Cámara de C. I. } \\
\text { de Torrelavega }\end{array}$ \\
\hline Castilla y León & $\begin{array}{l}\text { - SEPES } \\
\text { - CEPE } \\
\text { - APTE }\end{array}$ & $\begin{array}{l}\text { - ADE Parques Tec- } \\
\text { nológicos y Empre- } \\
\text { sariales (Gesturcal) }\end{array}$ & $\begin{array}{l}\text { Cámaras de Comer- } \\
\text { cio e Industria }\end{array}$ & - Ayuntamientos \\
\hline Galicia & $\begin{array}{l}\text { - SEPES } \\
\text { - CEPE } \\
\text { - APTE }\end{array}$ & $\begin{array}{l}\text { - IGVS/XESTUR } \\
\text { - SEA } \\
\text { (Suelo Empresarial } \\
\text { del Atlántico) }\end{array}$ & $\begin{array}{l}\text { - URBA Ourense } \\
\text { - Cámaras de } \\
\text { Comercio e } \\
\text { Industria (Lugo, } \\
\text { Ourense) } \\
\text { - SUPLUSA (Lugo) } \\
\text { - IPESPO } \\
\text { (Pontevedra) }\end{array}$ & $\begin{array}{l}\text { - Ayuntamientos } \\
\text { - Consorcio Zona } \\
\text { Franca de Vigo } \\
\text { - PLISAN } \\
\text { - Cámaras de C.I. } \\
\text { de Santiago, } \\
\text { Ferrol, A Coruña, } \\
\text { Tuy, Vigo, } \\
\text { Pontevedra, } \\
\text { Vilagarcía }\end{array}$ \\
\hline Navarra & $\begin{array}{l}\text { - SEPES } \\
\text { - CEPE }\end{array}$ & $\begin{array}{l}\text { - SODENA } \\
\text { - NASUINSA (Nava- } \\
\text { rra de Suelo Indus- } \\
\text { trial SA) }\end{array}$ & $\begin{array}{l}\text { - Cámara Navarra } \\
\text { de Comercio e } \\
\text { Industria }\end{array}$ & - Ayuntamientos \\
\hline País Vasco & $\begin{array}{l}\text { - SEPES } \\
\text { - SEPIDES } \\
\text { - CEPE } \\
\text { - APTE }\end{array}$ & $\begin{array}{l}\text { - SPRI } \\
\text { - SPRILUR }\end{array}$ & $\begin{array}{l}\text { - Diputaciones } \\
\text { Forales de Bizkaia, } \\
\text { Gipuzkoa y Álava }\end{array}$ & $\begin{array}{l}\text { - Ayuntamientos } \\
\text { - Puerto de Bilbao } \\
\text { - Sociedades } \\
\text { Industrialdeak }\end{array}$ \\
\hline
\end{tabular}

Fuente: Elaboración propia. 
El análisis de la distribución y localización de las áreas empresariales requiere, en primer lugar, disponer de una Base de Datos Bibliográfica y Documental, herramienta que es preciso generar mediante técnicas convencionales. El trabajo empieza por revisar la bibliografía disponible sobre suelo industrial y áreas empresariales e identificar/seleccionar las publicaciones y trabajos que pueden representar una aportación útil, tanto desde el punto de vista teórico como aplicado y práctico. La información obtenida se organiza de manera que permita búsquedas rápidas y sencillas. En paralelo, se rastrea la normativa y la legislación sobre Ordenación del Territorio, se identifican y reúnen en un listado los organismos e instituciones clave en materia de suelo industrial para cada Comunidad Autónoma y con el resultado se nutre esta Base de Datos primaria. Hay que añadir otra tarea clave que aporta información muy valiosa: localizar los planes oficiales de suelo industrial, no siempre publicados o disponibles en la Red, y añadirlos a la Base, sabiendo que cada Gobierno autonómico ha seguido pautas diferentes en cuanto a la fecha de aprobación, periodicidad y alcance de este instrumento generador de suelo público (se trata de una herramienta de política territorial/sectorial que algunas Comunidades tienen desde principios de los años de 1990 y que renuevan cada cuatro años, mientras que otras son menos regulares o puede que ni siquiera dispongan de un plan de suelo industrial o similar). También hay que localizar y volcar, en su caso, la información de organismos tales como Diputaciones, Zona Franca, Cámaras de Comercio e Industria, etcétera.

La Base de Datos primaria se complementa con otras dos bases específicas: la Base de Datos sobre Criterios de Ordenación de espacios empresariales y la Base de Datos sobre Suelo Industrial. La primera se alimenta con datos e información que se obtienen del examen pormenorizado de cada documento de Directrices Regionales de Ordenación del Territorio (y la correspondiente Ley que lo produce), cuyo enfoque y contenidos varían de unas comunidades autónomas a otras, pero que en general permiten conocer lo siguiente:

Principios generales de la ordenación del territorio para espacios empresariales

Criterios y objetivos sobre áreas empresariales y suelo industrial

Indicaciones específicas sobre suelo industrial y empresarial: instrumentos de actuación, tipología de espacios a ordenar y localizaciones preferentes.

Con esta información sistematizada es posible realizar análisis y alcanzar conclusiones sobre la estrategia de los poderes públicos en relación con la 
promoción económica y la cohesión territorial dentro de cada Comunidad Autónoma, identificando las situaciones de equilibrio y/o desequilibrio territorial sobre las que se trata de intervenir y el alcance de las acciones concretas que se proponen para cohesionar el espacio regional. Este recurso también permite comparar estrategias entre regiones y valorar el papel que desempeñan en cada caso elementos tales como las infraestructuras de transportes y comunicaciones o la red urbana.

Por último, a partir de los criterios y propuestas sobre ordenación de los espacios económicos es posible deducir las claves del modelo territorial por el que apuesta cada Gobierno autonómico, lo que a su vez está condicionado por la política sectorial subyacente, pues hay que tener presente que las acciones en materia territorial dependen en gran medida de los objetivos de la política económica, de ahí que, por ejemplo, en coyunturas de recesión o crisis económica se frene la expansión o creación de nuevas áreas empresariales y con ello se ponga límites a la tendencia a la dispersión o cierta difusión territorial de las actividades productivas.

Más complicado es obtener una representación espacial o cartografía del conjunto de las propuestas para ámbitos suprarregionales, el norte de España en nuestro caso, pues las Directrices de Ordenación del Territorio solo ofrecen, y no siempre, esquemas de las acciones propuestas, sin escala ni referencias geográficas precisas (figura 1). Esta carencia obliga a representar de forma orientativa los espacios y lugares señalados por cada normativa autonómica para el desarrollo de suelo de uso empresarial, lo que aporta una imagen sintética y expresiva de la magnitud y distribución espacial de las áreas empresariales previstas, pero imprecisa.

Por su parte, la Base de Datos sobre Suelo Industrial se nutre de información sobre polígonos industriales, parques empresariales y parques científicos y tecnológicos obtenida mediante trabajo de campo y contraste de datos procedentes de fuentes diversas facilitadas por las CCAA, los Ayuntamientos, Diputaciones $y$, en general, entes privados y públicos promotores y/o gestores de suelo empresarial (CEPE, APTE, asociaciones de polígonos, organizaciones de empresarios...). La principal fuente de información son los planes oficiales de suelo industrial vigentes en cada Comunidad Autónoma. Donde este instrumento no existe (Castilla y León, por ejemplo) hay que identificar su equivalente. Se trata, como antes se ha dicho, de un instrumento de política territorial-sectorial competencia de las consejerías de Industria, Ordenación del Territorio y Urbanismo y que con frecuencia gestionan las agencias de desarrollo regional o algún organismo de suelo creado a tal fin (p.ej. Sprilur en el País Vasco, creado para garantizar la existencia de suelo público y de pabello- 


\section{FIGURA 1}

\section{LOCALIZACIÓN ESQUEMÁTICA DE LAS ÁREAS EMPRESARIALES A DESARROLLAR EN GALICIA SEGÚN LAS DOT (2011)}

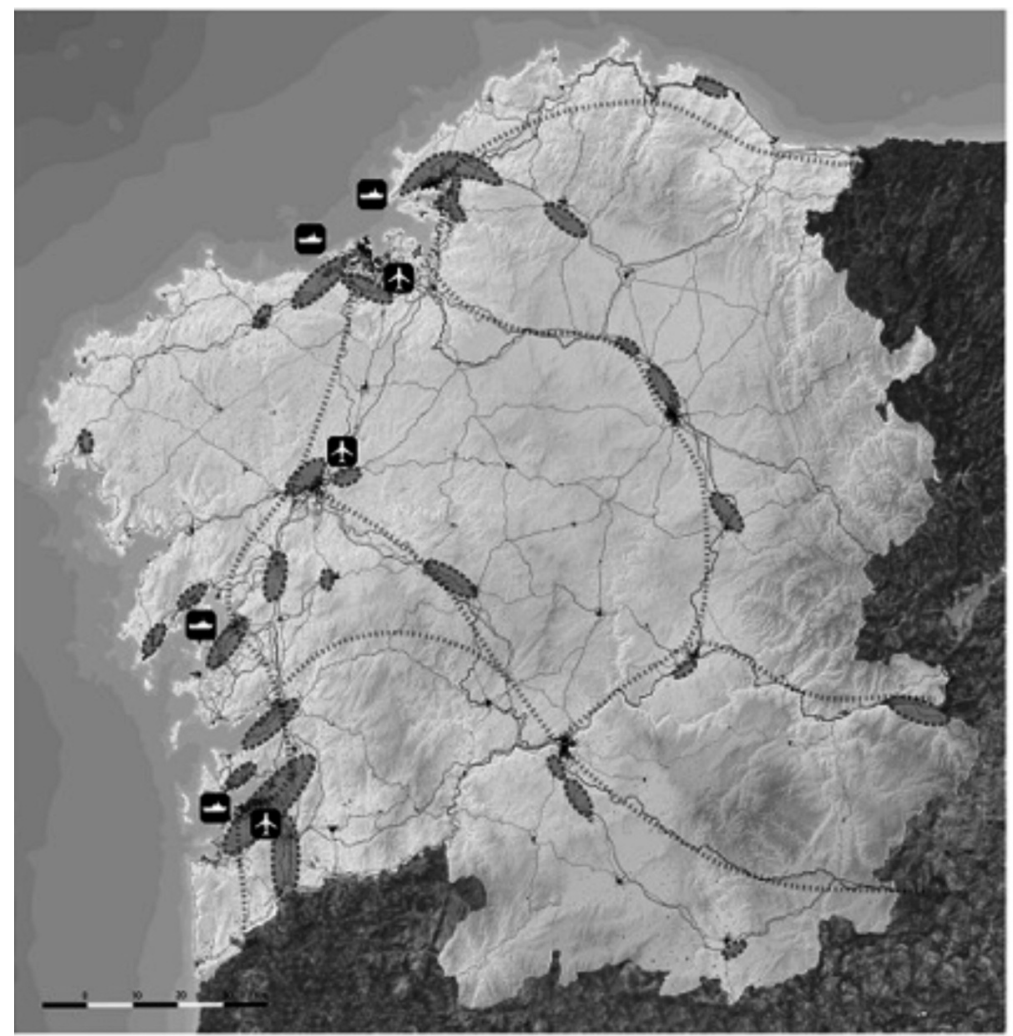

Fuente: Xunta de Galicia, DOT 2011.

nes en cantidad y calidad suficientes para atender la demanda y fomentar la actividad industrial y empresarial como fuente de empleo y de riqueza, según se enuncia en la fuente oficial http://www.sprilur.es/grupo-sprilur/ (Verificada el 22/03/2015).

También la Base sobre Suelo Industrial se alimenta con datos obtenidos de los planes de suelo empresarial para ámbitos territoriales de escala infra-regional (caso de Castilla y León y de Navarra). Especial referencia merece la Base de Datos facilitada por CEPE, que recoge información sobre un amplio nú- 
mero de áreas empresariales en España agrupadas por CCAA, con información de cada polígono industrial o parque empresarial, aunque presenta debilidades: no es exhaustiva, está muy incompleta en algunas CCAA, no está geo-referenciada y se cierra en 2008.

En suma, la operación de investigación descrita permite organizar y sistematizar los datos básicos sobre suelo industrial de promoción pública y privada, esto es: superficie, localización, modalidad de asentamiento, promotor, ventas de parcelas, ocupación empresarial, etcétera. Con esta herramienta el análisis se profundiza ya que permite conocer con detalle los desarrollos de suelo industrial/empresarial y su evolución en la última década, comparar el proceso entre Comunidades Autónomas y contrastar situaciones locales (rural/urbano) para alcanzar algunas conclusiones de validez general.

Llegados a este punto se plantea la necesidad de disponer de una cartografía adecuada que dé cuenta de la distribución real y la localización precisa de las áreas empresariales. A tal efecto parece conveniente que los datos cartográficos se refieran a información territorial asociada a polígonos. De las distintas fuentes de datos existentes se ha optado por la que elabora y distribuye el Instituto Geográfico Nacional (IGN) a través de su Centro de descargas para uso no comercial.

Una vez consultado el catálogo de productos del Centro de descargas del CNIG y tras una serie de consideraciones, entre ellas la superficie total objeto de nuestro estudio (unos $157.346 \mathrm{~km}^{2}$ ), optamos por la Base Cartográfica Numérica a escala 1:200.000 (BCN 200) como fuente de datos principal. Dicha Base es un conjunto de datos geográficos vectoriales obtenido por digitalización y edición de la información originalmente contenida en la serie de mapas provinciales a escala 1:200.000 del IGN, posteriormente enriquecida y actualizada mediante la generalización de los datos de la BCN 25. La información vector resultante se contrastó con otras fuentes de información, en especial con las imágenes SPOT con resolución de 2,5m, y mediante la aplicación de las especificaciones de BCN200 conseguimos un producto con información geográfica estructurada por temas y actualizada al año 2010. Este recurso facilitado por el CNIG va destinado a todo tipo de usuarios (empresas, Administraciones públicas, etcétera), para todo tipo de aplicaciones y sobre todo tipo de plataformas. Se trata de un sistema de información geográfica multipropósito que alberga datos geográficos y temáticos y es capaz de servir de soporte tanto a consultas geográficas como a la creación de diversos productos cartográficos, además de ser una referencia en otros sistemas de información geográfica. La actual BCN 200 tiene como características 
fundamentales el ser un SIG continuo (no dividido por hojas), la existencia de una tabla por cada fenómeno geográfico y una geometría explicita (punto, línea, área).

Para el objetivo que aquí nos ocupa se realizó la descarga seleccionando como unidad la provincia. Toda la información descargada por provincias servirá, a su vez, para componer los distintos mapas que interesan a efectos de distribución, localización y articulación territorial de áreas empresariales.

\section{DISEÑO DE LA BASE DE DATOS CARTOGRÁFICA DE ÁREAS EMPRESARIALES}

En el ámbito de los SIG domina una oferta considerable de datos, los cuales deben ser ingresados, clasificados, procesados, analizados y distribuidos de una manera rápida y eficiente. Los datos geográficos se prestan a ser manipulados con la ayuda de bases de datos. La «base de datos espacial» que hemos generado muestra información de las áreas empresariales del norte de España procedente de diversos censos y listados y volcada en la Base de Datos sobre Suelo Industrial descrita en el apartado anterior. A partir de aquí creamos una Base de Datos Cartográfica aplicando el concepto de «área empresarial».

Por áreas empresariales entendemos los terrenos delimitados y desarrollados como espacios urbanizados para la industria y otras actividades económicas en modalidades de asentamientos que se denominan «polígonos industriales», «parques industriales», "parques empresariales» y "parques científicos y tecnológicos», ocupados por empresas de muy diferente tamaño y dedicadas a actividades de fabricación y/o servicios a la industria y a otras empresas, lo que incluye con frecuencia cierto comercio de mercancías diversas e incluso actividades de distribución (es frecuente que en los polígonos industriales tomen asiento plataformas logísticas, como se observa, por ejemplo, en los polígonos leoneses de Onzonilla-León II (Inditex) y de Villadangos (Mercadona). Se excluyen las localizaciones industriales aisladas, sea cual fuere el tamaño de las instalaciones.

El diseño y puesta en marcha de una base de datos geo-referenciada sólida y eficiente es un requisito indispensable para lograr un SIG exitoso. En nuestro caso el diseño de la base de datos espacial propuesta se basa en los productos de ESRI (ArcGis) que utilizan un SGBD (Sistema Gestor de Bases de Datos) de estructura de datos relacional y con formato de salida vectorial, como ya se ha mencionado. Además, el diseño y elaboración de la capa base 
supone el mayor esfuerzo total del proyecto SIG sobre las áreas empresariales del norte de España.

La metodología utilizada en la creación de la Base de Datos Cartográfica distingue cuatro etapas principales dentro de un procedimiento típico al crear bases de datos espaciales utilizando herramientas SIG:

1. Recopilación y entrada de datos. Esta tarea consiste en la ubicación, identificación y adquisición de toda la información disponible, necesaria para desarrollar la base de datos espacial sobre áreas empresariales. El éxito en la creación de la base de datos depende en buena medida de esta tarea preliminar, ya que conocer las características de los datos, la información respecto al origen, los métodos de elaboración o transformación, el propósito, su fecha, etcétera, es tan importante como los propios datos en sí para el trabajo posterior con ellos. Los datos pueden estar disponibles en distintos formatos (papel, archivos informáticos, imágenes) o pueden no estar disponibles o ser inexistentes, lo que obliga a la tarea de generarlos con distintas técnicas (trabajo de campo, fotointerpretación y teledetección de imágenes, entre otras). Una vez realizada la identificación de toda la información pertinente, es necesario evaluarla en función de la utilidad para nuestros objetivos. Los datos proceden de dos fuentes ya citadas aquí: la primera es la Base Cartográfica Numérica a escala 1:200.000 (BCN 200) del Instituto Geográfico Nacional, 2010; y la segunda es la Base de Datos sobre Suelo Industrial arriba descrita. En esta etapa se trata también de incorporar al sistema de una manera lógica y ordenada toda la información obtenida con destino a la Base de Datos Cartográfica.

2. Procesamiento preliminar de los datos geoespaciales. En esta fase se realiza el tratamiento de toda la información recopilada para que pueda ser ingresada apropiadamente al SIG. Se llevan a cabo tres acciones principales: primero, la selección y extracción de la información referida al uso industrial de la capa de usos del BCN200 (formato shp) del IGN por ámbitos provinciales de la zona de estudio; segundo, la integración de la información anterior con la Base de Datos sobre Suelo Industrial (formato excel) que hemos generado; y tercero, nuestra aportación final con la información obtenida en el trabajo de campo sobre áreas empresariales no registradas (o improcedentes) en las fuentes mencionadas, lo que conllevará la digitalización de nuevas áreas empresariales. Una vez procesada toda la información se concreta simplificando los registros disponibles en la base de datos según el resultado 
que se pretende alcanzar. Campos finales que aparecerán en la misma: ID, Nombre del área empresarial, Tipología de asentamiento, Comunidad Autónoma, Provincia, Municipio, Localidad, Tipo Promoción, Promotor y Superficie en hectáreas. Es en esta etapa donde se concentra el mayor esfuerzo de trabajo, integrando multitud de registros. Esta exhaustiva tarea consiste en consultar una por una más de 2.300 entradas o polígonos referidos al uso industrial, para editar y etiquetar exhaustivamente (nombre común de la delimitación de la zona o polígono, el 95\% de las áreas en la capa de origen del IGN vienen sin etiquetar) y conocer así cual es la característica fundamental de cada espacio. Esta tarea se lleva a cabo para todas las provincias de las seis Comunidades Autónomas de nuestro ámbito de estudio, en total unas 2.362 áreas o zonas empresariales.

3. Manejo y administración de la información. Etapa relacionada con los procedimientos de creación y acceso a los diferentes componentes de la base de datos, creando métodos sencillos para captura, actualización, consulta y eliminación de la información residente en la base construida hasta el momento. Se establece la coordinación entre el equipo generador de los datos, el administrador del sistema y operador analista principal del SIG (único con acceso de lectura y escritura), y la dirección del proyecto. Esta coordinación permite una recolección, actualización y eliminación de datos que garantiza la integridad y continuidad en la base de datos. Es aquí cuando se depuran o eliminan las entidades o áreas, ahora etiquetadas, no referidas a espacios urbanizados para la industria u otras actividades económicas, pero que sí aparecían en la capa de usos del BCN200 del IGN como de uso industrial (fábricas u otras formas de actividad industrial aisladas). El total de áreas empresariales delimitadas aparecen en el cuadro 2.

4. Procesamiento, análisis de los datos y generación de productos. Es una de las etapas más productivas y tiene como objetivo principal la generación de nueva información basándose en los datos existentes en la base de datos generada. Se trata de generar información espacial que permita observar la realidad de los espacios industriales existentes en el ámbito de estudio para conseguir visualizar resultados y analizar las dinámicas territoriales respecto a este factor en las distintas zonas de estudio. En definitiva, se trata de crear un sistema que pretende proveer y aumentar la cantidad y la calidad de la información y facilitar así el análisis y la toma de decisiones sobre las áreas empresariales, un elemento a su vez relacionado con otros componentes espaciales. 


\section{CuAdro 2}

AGENTES PROMOTORES Y GESTORES DE ÁREASEMPRESARIALES EN EL NORTE DE ESPAÑA

\begin{tabular}{lccc}
\hline \multicolumn{1}{c}{ CCAA } & $\begin{array}{c}\mathbf{N}^{\text {o }} \text { áreas } \\
\text { empresariales }\end{array}$ & $\begin{array}{c}\text { Superficie de suelo } \\
\text { industrial }(\mathrm{Km} 2)\end{array}$ & $\begin{array}{c}\text { Superficie de suelo } \\
\text { industrial }(\mathrm{Km} 2)\end{array}$ \\
\hline País Vasco & 350 & 58,44 & 0,80 \\
Navarra & 150 & 35,55 & 0,34 \\
Galicia & 226 & 83,47 & 0,28 \\
Castilla y León & 514 & 128,56 & 0,13 \\
Cantabria & 80 & 17,71 & 0,33 \\
Asturias & 136 & 28,60 & 0,26 \\
\hline Total & 1456 & 352,33 & 0,22 \\
\hline
\end{tabular}

Fuente: Elaboración propia.

En definitiva, las principales acciones a ejecutar con la puesta en marcha del SIG descrito serían las siguientes:

- Localizar las áreas empresariales del norte de España.

- Medir longitudes perimetrales y calcular superficies de esas zonas, pudiendo obtener totales por municipios, provincias, CCAA y para el total del ámbito territorial considerado.

- Digitalizar nuevos espacios industriales en la zona de estudio.

- Realizar operaciones de edición sobre superficies industriales existentes (agregación, segregación, modificación de los vértices de áreas ajustándolas a la realidad, etcétera).

- Realizar mapas de situación de áreas empresariales concretas.

- Realizar estudios comparativos de áreas empresariales según su tipología, tamaño, promotor, grado de consolidación, etcétera, identificando visualmente en colores diferentes los grupos con características similares.

\section{HERRAMIENTAS CARTOGRÁFICAS DESARROLLADAS Y SU UTILIDAD}

Actualmente se dispone de multitud de técnicas para la representación de los datos y entre ellas destaca la elaboración de mapas o cartografía con SIG, es decir, la producción de mapas por medios digitales con la información de 
las bases de datos. Como ya sabemos, el trabajo cartográfico es inherente al estudio geográfico y al análisis del territorio porque permite la aproximación a diferentes ámbitos espaciales (local, regional, nacional) y permite también seleccionar y simplificar variables significativas. Las sálidas cartográficas suele ser el último paso en el proceso de un proyecto GIS y es importante, aunque tengamos unos buenos datos espaciales y la información sea muy precisa, incidir en el diseño y su composición, ya que un mapa visualmente confuso no es útil a ningún efecto. Las formas para representar la cartografía han aumentado de manera notable coincidiendo con el desarrollo de las nuevas tecnologías y son multiples los factores a tener en cuenta a la hora de elaborarla: resolución, tamaño, modelos, colores, tiempo de producción, etcétera.

Las herramientas de trabajo utilizadas para el geoprocesamiento de la información han sido distintos softwares SIG. Como se ha comentado, los SIG permiten ingresar, almacenar, editar y analizar datos geográficos. El proposito es convertir esos datos en información detallada para el análisis y la toma de decisones sobre la estructura y las dinámicas de suelo empresarial. Es decir, realizar una cartografia capaz de representar en mapas la distribución espacial de las áreas empresariales en el norte de España a distintas escalas y con distintas simbologías. Esto se consigue porque los SIG permiten presentar la información obtenida en forma de mapas. El tratamiento cartográfico una vez creada la capa de Areas Industriales del Norte de España, será facilitar la edición de un atlas de áreas empresariales, un conjunto de fichas descriptivas de los polígonos, así como todo tipo de cartografía temática con distintas escalas.

Para la realización de la cartografía temática optamos, tal y como se ha indicado más arriba, por la Base Cartográfica Numérica a escala 1:200.000 (BCN 200) como fuente de datos principal y base cartográfica para la elaboración de la mayoría de los mapas. Como complemento a esta cartografía base se han utilizado otras fuentes de información a fin de contrastar y actualizar la capa base sobre suelo industrial en todo el área de estudio, como han sido los servicios WMS del PNOA (Plan Nacional de Ortofotografía Aérea) y de la Oficina Virtual del Catastro. Además, se han rastreado y analizado los directorios online de suelo industrial disponibles para nuestro ámbito de análisis, un recurso que antes estaba disponible en papel, y era fácil hallar en bibliotecas y hemerotecas, pero que hoy se edita mayoritariamente en la Red, donde se encuentran las versiones más actualizadas. Se trata, no obstante, de una fuente con limitaciones, pues estos directorios carecen de fecha de edición y se desconoce si hay una regularidad en su elaboración.

Por otra parte, dado el componente territorial del suelo empresarial es importante ofrecer, además de información alfanumérica y bibliográfica, una in- 
formación espacial lo más ajustada y completa posible. Por ello, y a modo de conclusión, se muestran algunos ejemplos de la cartografía a diferentes escalas obtenida sobre áreas empresariales en el norte de España con las herramientas y la metodología descritas. Se trata de imágenes inéditas y de alto valor descriptivo y analítico que permitirán avanzar en el conocimiento de los espacios industriales, su lógica espacial y su estructura territorial. Un esfuerzo aún en fase embrionaria, pero que permite vislumbrar el modelo territorial que resulta de la combinación de políticas públicas e intereses privados y de las condiciones de cada territorio respecto a su proceso histórico de industrialización, el impacto de las sucesivas crisis industriales, el dinamismo actual de la industria y las características del tejido empresarial. En la cartografía que aquí se muestra se recoge la totalidad de suelo industrial existente en las seis comunidades objeto de estudio, abarcando el amplio abanico de tipologías que lo conforman: polígonos industriales, parques industriales y empresariales, parques tecnológicos o simplemente una zona industrial así calificada por el planeamiento urbanístico. Sin pretender de momento hacer ninguna distinción en cuanto a su grado de desarrollo.

El desarrollo del Geovisor o visor cartográfico que muestra la figura 2 ofrece la posibilidad de hacer búsquedas territoriales: por municipios, provin-

FIGURA 2

GEOVISOR DESARROLLADO CON HERRAMIENTAS

DE SOFTWARE LIBRE, OPENGEOSUITE

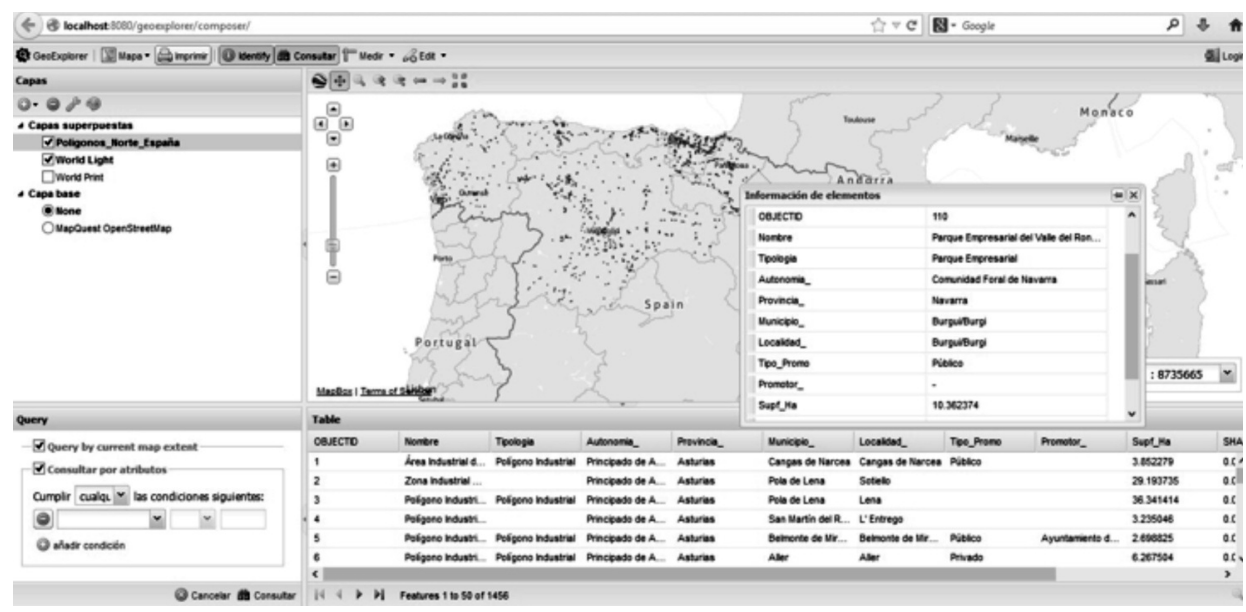

Fuente: Elaboración propia.

Estudios Geográficos, Vol. LXXVI, 278, pp. 39-62, enero-junio 2015 ISSN: 0014-1496, eISSN: 1988-8546, doi: 10.3989/estgeogr.201502 
cias, Comunidades Autónomas, por superficie, tipología o nombre del espacio productivo, geo-referenciando los elementos mostrados. Con esta herramienta se puede navegar por el mapa y hacer zoom, así como seleccionar los espacios industriales para obtener información de los mismos. Asimismo, permite activar o desactivar capas de información y visualizar la tabla alfanumérica. Acciones todas ellas de gran ayuda en el análisis de la estructura territorial de la industria y los tejidos empresariales a distintas escalas, permitiendo la comparación y contraste entre territorios y lugares donde están ubicadas áreas empresariales de diferente tamaño y tipología.

Uno de los logros más interesantes de nuestra propuesta metodológica, a través de las herramientas y recursos descritos, tiene reflejo en la figura 3. En ella se ofrece una imagen inédita de la localización y distribución real y precisa de las áreas empresariales en el norte de España, un aspecto clave para comprender y explicar el modelo territorial de la industria, teniendo en cuenta elementos fundamentales como son la presencia de grandes ciudades, áreas urbanas polinucleares o núcleos rurales de cierta entidad (lo que remite a la relación entre industria y ciudad y las pautas que en la actualidad rigen el comportamiento espacial de la industria y demás actividades productivas, proceso a su vez conectado con la toma de decisiones por parte del empresariado

FIGURA 3

\section{DISTRIBUCIÓN Y LOCALIZACIÓN DE LAS ÁREAS EMPRESARIALES EN EL NORTE DE ESPAÑA, 2012}

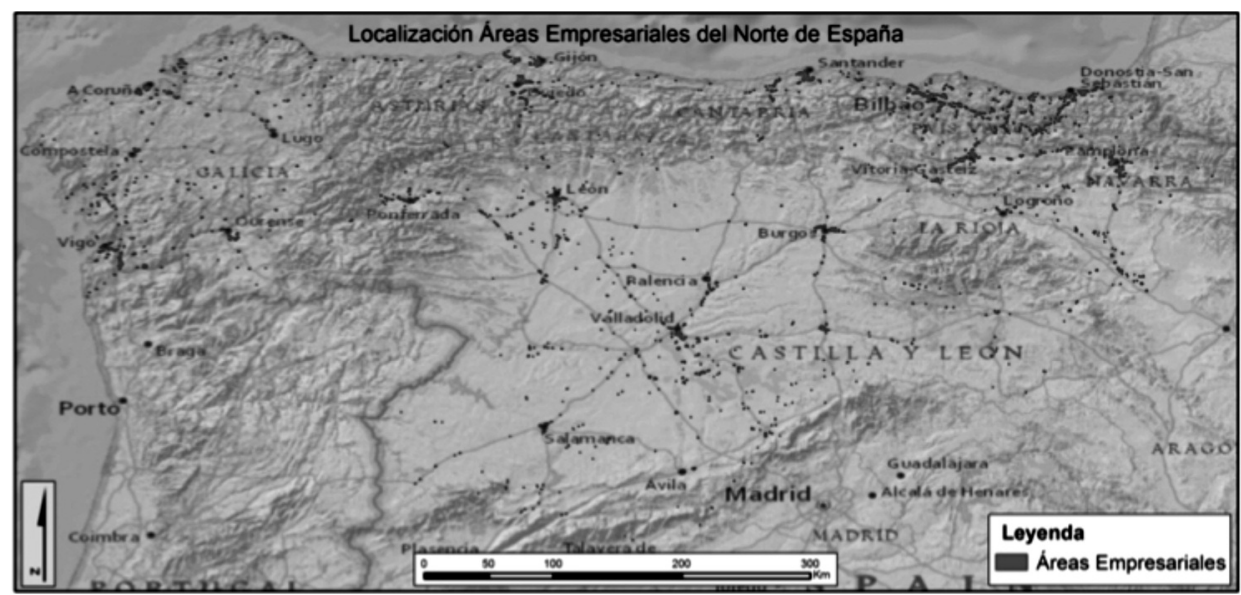

Fuente: Elaboración propia. 
y con el alcance de las políticas sectoriales y territoriales) y la red de infraestructuras de transporte y comunicaciones.

El mapa de la figura 3 es expresivo, en efecto, del papel que en la localización de las áreas empresariales desempeñan la disposición y desarrollo de las vías de transportes y comunicaciones, incluidos los puertos de mar, tan decisivos en el proceso histórico de industrialización de la zona atlántica y aún hoy decisivos para ciertas actividades productivas y logísticas (por ejemplo, el desarrollo de la ZALIA en Gijón así lo demuestra). En la imagen se aprecian concentraciones en torno a las áreas urbanas consolidadas, las ciudades de mayor tamaño del sistema urbano, aunque también se perfila el creciente protagonismo de las ciudades medias y el esbozo de corredores industriales, del tipo que ilustra la figura 4.

FIGURA 4

CARTOGRAFÍA PARA REPRESENTAR LAS ÁREAS EMPRESARIALES EN RELACIÓN CON LAS ZONAS URBANAS Y LAS INFRAESTRUCTURAS

DE TRANSPORTES, 2012

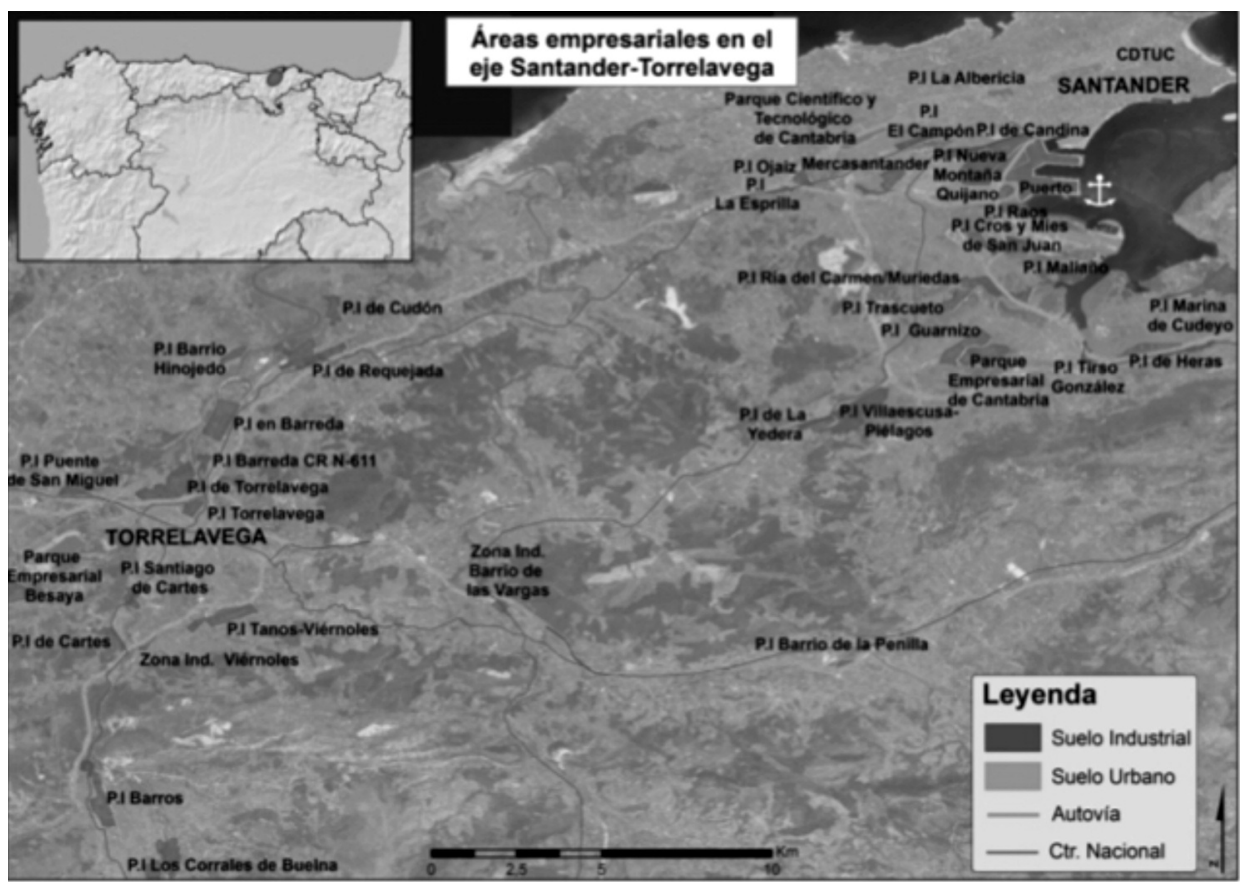

Fuente: Elaboración propia.

Estudios Geográficos, Vol. LXXVI, 278, pp. 39-62, enero-junio 2015 ISSN: 0014-1496, eISSN: 1988-8546, doi: 10.3989/estgeogr.201502 
Incluso se puede profundizar en la evolución del modelo territorial de la industria comparando nuestro mapa con un mapa industrial histórico: a grandes rasgos se confirma la tendencia dominante a la concentración de las actividades y áreas productivas en zonas de antigua industrialización (ría de Bilbao, bahía de Santander, el Ocho asturiano, el eje Ferrol-Vigo), pero con matices que perfilan el modelo distributivo actual, añadiendo una cierta difusión, rasgo que a su vez lo asimila a otros modelos europeos (Francia, Alemania, Bélgica). Por último, es apreciable un cierto efecto frontera entre unas CC AA y otras, fenómeno que se explica por el impacto, precisamente, de las políticas públicas.

A escala urbana la herramienta desarrollada también ofrece amplias posibilidades para el análisis y comprensión de los fenómenos territoriales relacionados con la industria. Un ejemplo de aplicación lo tenemos en Gijón (Asturias): la figura 5 ilustra la distribución de la áreas empresariales en la ciudad y evidencia la concentración de polígonos industriales, parques empresariales y zona logística en el sector Oeste, en relación directa con el puerto marítimo del Musel y las instalaciones siderúrgicas de la empresa Arcelor-Mittal (antigua Ensidesa), que en un pasado reciente aglutinaron a su alrededor a las empresas del sector metalmecánico, propiciando la formación del complejo industrial-portuario de Gijón. Este modelo distributivo, altamente concentrado, puede explicarse a la luz del cruce o superposición de las acciones históricas de promoción de suelo industrial protagonizadas por dos instancias públicas: el Gobierno del Principado de Asturias y el Ayuntamiento de Gijón.

El crecimiento de la oferta de suelo industrial en Gijón es constante desde los años de 1960, siendo la iniciativa privada la que protagoniza el desarrollo de los primeros polígonos industriales en terrenos destinados a uso industrial por el planeamiento urbanístico. A partir de 1984 se abre una etapa de creación de oferta pública que se prolonga hasta la actualidad, siendo sobresaliente la iniciativa municipal, que puntualmente se ve reforzada por la intervención del Gobierno del Principado de Asturias, que impulsa diversos planes regionales de suelo industrial para responder a la demanda de los empresarios (claro exponente de la política sectorial que convierte el suelo en un recurso para atraer y fijar la inversión).

La vigencia del llamado Polo de Desarrollo de Oviedo, instrumento de política industrial de la etapa del desarrollismo franquista, sirvió de impulso a los primeros polígonos privados, que se concentran, como se ha dicho, en el Oeste de la ciudad y tienden a ocupar terrenos suburbiales y periurbanos próximos a los barrios portuarios, la zona de astilleros o en espacios libres de usos residenciales próximos a la factoría siderúrgica de Veriña. De esta época son los polígonos hoy consolidados de Bankunión I (1965), Bankunión II 
(1968), Promosa (1969) y el origen del área industrial de la Juvería (1969). Otra pequeña agrupación industrial toma cuerpo de forma espontánea en los años setenta, pero en 1987 se convierte en el polígono de Maximino Vega. La intervención municipal en materia de suelo industrial se produce, sobre todo, a partir de los años ochenta: las primeras intervenciones se localizan en Tremañes, donde el Ayuntamiento desarrolla el polígono de Mora de Garay (1987); en Roces, donde crea cinco polígonos colindantes entre sí (a partir de 1984); y en Porceyo, con un polígono que comienza a urbanizarse en 1991. La operación urbanística sobre Roces-Porceyo logró introducir cierto orden en una zona de industrialización espontánea y caótica situada en la periferia Sur de la ciudad desde los años setenta del pasado siglo Xx. En una etapa posterior el Ayuntamiento de Gijón expropió terrenos a Ensidesa para crear un polígono industrial desarrollado por fases desde 1995: Somonte I-II y Somonte III. También a finales de los noventa la iniciativa municipal construye el llamado polígono de Tremañes-Centro de Transportes.

\section{LOCALIZACIÓN DE LAS ÁREAS EMPRESARIALES EN LA CIUDAD DE GIJÓN (ASTURIAS), 2012}

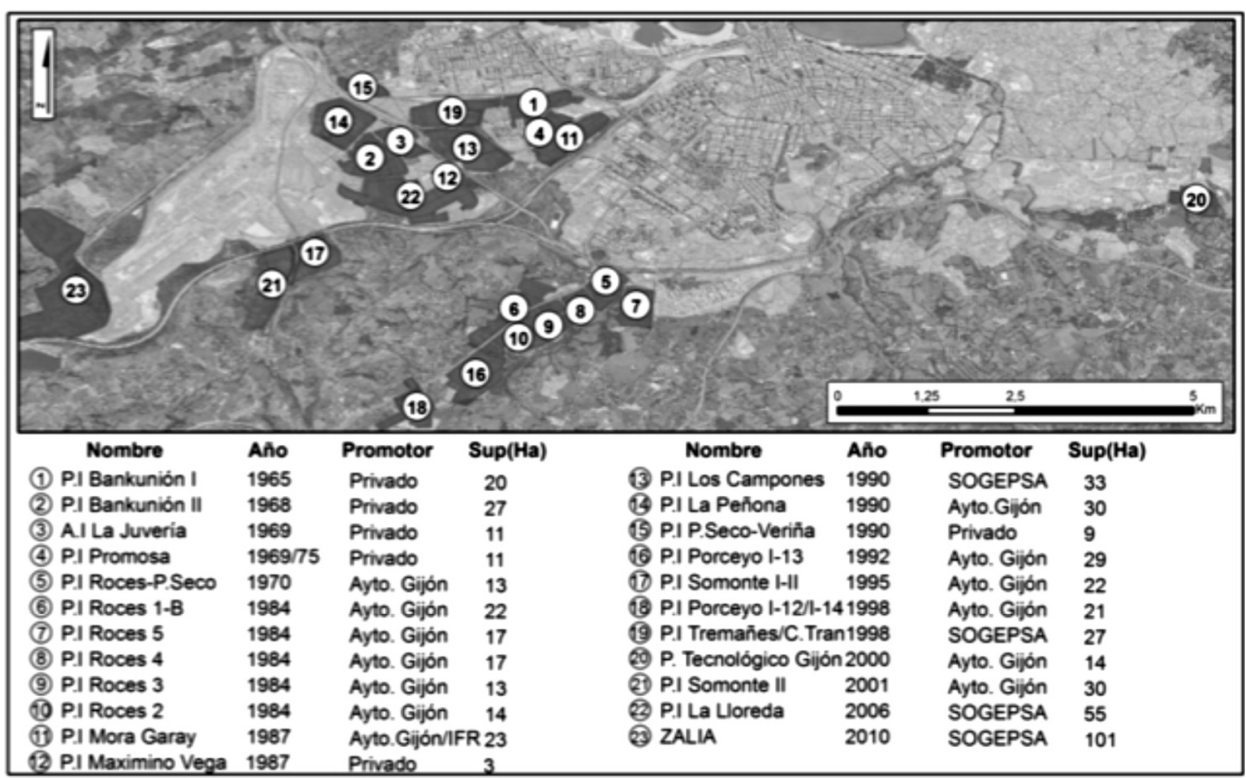

Fuente: Elaboración propia. 
Por su parte, la intervención del Principado de Asturias a través de los planes de suelo industrial regional se ha traducido en más suelo público para Gijón. Así el Plan oficial de 1990-1993 propuso la ampliación de Roces-Porceyo en 19 hectáreas; el Plan de suelo de 2001-2004 incluyó el polígono de LLoreda-Tremañes con 80 hectáreas, más 20 hectáreas para ampliar Somonte y otras cinco hectáreas para el nuevo Parque Científico y Tecnológico, situado en Cabueñes, una zona residencial de calidad sin presencia de la industria y, en tal sensido, una localización excepcional y fuera del «mapa industrial» gijonés. Por su parte, el Plan 2005-2008 retomó el polígono de Lloreda, herencia del plan anterior, que también será recogido en el plan vigente 2009-2012, que aprobó ampliar el Parque Científico y Tecnológico con casi cuatro hectáreas añadidas a las ya consolidadas y asumió el seguimiento del desarrollo de las 98 hectáreas brutas del polígono industrial de Lloreda, la intervención más ambiciosa en la ciudad.

Gijón dispone en la actualidad de unas 583 unas de suelo empresarial fragmentado en unidades pequeñas que van tejiendo una trama densa de naves, fábricas y talleres en el sector occidental del plano urbano resultado de un proceso histórico de industrialización que se refuerza en los años del industrialismo, con el protagonismo de la gran fábrica, para evolucionar hacia un espacio industrial de asentamientos planificados de iniciativa pública, tendencia extrapolable a la mayoría de las ciudades del norte peninsular (Fernández y Quirós, 2010; Benito 2011; Humbert y otros, 2011 ).

En suma, el caso empírico de la ciudad asturiana nos ha permitido ilustrar el potencial de la herramienta que aquí se describe así como su valor para el análisis urbano del suelo empresarial, no solo en términos estructurales, sino también dinámicos pues a partir de una imagen estática es posible, recurriendo a la historia económica y a la documentación y literatura disponible, explicar el proceso de consolidación de los asentamientos industriales. Asimismo hay que subrayar que tal herramienta es un recurso versátil y de amplio espectro, ofreciendo una pauta para el estudio comparativo a partir de criterios homogéneos.

\section{CONCLUSIONES}

La metodología de estudio de las áreas empresariales requiere herramientas que es preciso desarrollar teniendo en cuenta las características y limitaciones de las fuentes de información disponibles, muy variadas y variopintas según los espacios regionales considerados, y, por esto mismo, difíciles de superponer de manera mecánica. Se hace necesario, con frecuencia, generar y sistema- 
tizar la información para un análisis que combine escalas (municipal, provincial, regional y urbana) y que permita matizar fenómenos y también extraer conclusiones o llegar a resultados con validez general, evitando la excepcionalidad o la mera descripción de casos inconexos. Por su parte, el tratamiento de los datos debe hacerse teniendo en cuenta los objetivos de la investigación y las características de las herramientas digitales a las que van a servir de nutriente, unas herramientas que se diseñan a partir de modelos disponibles y ensayados con demostrado rendimiento en otro tipo de trabajos, pero insuficientes en su configuración estándar para dar respuesta a la multitud de cuestiones que plantea el estudio geográfico las áreas empresariales.

En este artículo se ha intentado mostrar cómo crear sencillos instrumentos ad hoc para obtener un alto rendimiento de los datos y la información sobre áreas empresariales y suelo industrial y, a partir de aquí, llegar a comprender y explicar la complejidad de la lógica espacial de la producción, en la que intervienen numerosos agentes públicos y privados. El foco del estudio lo constituyen las áreas empresariales que forman parte del mapa industrial del norte peninsular, repartidas entre CC AA que aplican políticas sectoriales y territoriales ajustadas a planteamientos y criterios muy variados que se traducen en acciones generadoras de una oferta de suelo para las empresas cuya localización, desarrollo y consolidación hay que analizar en profundidad, lo que reclama representaciones a la altura de las tecnologías digitales disponibles para llegar a obtener una cartografía de apariencia convencional, pero altamente resolutiva y expresiva de fenómenos y procesos de difícil o nula lectura e interpretación a partir de los recursos habituales.

En definitiva, las técnicas y los instrumentos de análisis expuestos en este trabajo son un paso tentativo para avanzar en el conocimiento geográfico de los espacios productivos y, en particular, en la dinámica y estructura de las áreas empresariales españolas, que actualmente toman la forma de polígonos o parques empresariales y tecnológicos en sustitución de los complejos productivos articulados en torno a la gran fábrica del siglo XIX y primera mitad del xx. Hoy la industria y los servicios tienden a concentrarse según unos factores de localización que dependen, en gran medida, de las políticas públicas y la urbanización general del territorio, con un papel protagonista de la ciudad y los espacios de calidad, donde la eficacia de las infraestructuras de transportes y comunicaciones para reforzar la accesibilidad y conectar mercados es determinante, pues de ello depende la competitividad de los territorios.

Fecha de recepción: 30 de julio de 2013.

Fecha de aceptación: 9 de diciembre de 2014. 


\section{BIBLIOGRAFÍA Y FUENTES}

Benavent, M. (2006): La ordenación del territorio en España. Evolución del concepto y de su práctica en el siglo Xx. Sevilla, Universidad de Sevilla/Junta de Andalucía.

Benito del Pozo, P. (2006): "Promoción y valorización del suelo industrial en Asturias”. Boletín de la Asociación de Geógrafos Españoles, 42, pp. 99-120.

Benito del Pozo, P. (2010): "Ordenación y planificación regional de suelo industrial. Asturias y su entorno regional". Geographicalia, 58, pp. 57-79.

Benito del Pozo, P. (dir.) (2011): Áreas empresariales, suelo industrial y logística: análisis y procesos en el territorio. Navarra, Aranzadi-Thomson Reuters.

Bielza de Ory, V.; Escolano, S. e Ibarra, P. (2010): De la ordenación a la planificación territorial estratégica en el ámbito regional-comarcal. Zaragoza, Prensas Universitarias de Zaragoza.

Bosque, J. y García, R. C. (2000): "El uso de los sistemas de información geográfica en la planificación territorial". Anales de Geografía de la Universidad Complutense, 20, pp. $49-67$

Bosque Sendra J. y Moreno Jimenéz, A. (2004): Sistemas de información geográfica y localización de instalaciones y equipamientos. Madrid, Editorial RA-MA.

Calderón, B. y Pascual, H. (2009): "Infraestructuras de soporte en los procesos de innovación regional: los nuevos espacios productivos en Castilla y León”. Boletín de la Asociación de Geógrafos Españoles, 49, pp. 237-254.

Carreras, C. y Ariño, A; Martinez, S. (2005): "Precisión y cambio de escala en la cartografía temática". Revista Mapping, 100, pp. 14-24.

CECODET (2000): El suelo industrial de Gijón. Gijón, Ayuntamiento de Gijón.

Directiva 2007/2/Ce del Parlamento Europeo y del Consejo de 14 de marzo de 2007 por la que se establece una infraestructura de información espacial en la Comunidad Europea (Inspire). DOUE del 25 de abril de 2007.

Esteban, M.; Álvarez, I. y Torres, C. (2012): "Políticas de suelo industrial en Álava". Boletín de la Asociación de Geógrafos Españoles, 58, pp. 133-156.

Feria, J. M.; García, A. y Ojeda, J. F. (eds.) (2009): Territorios, sociedades y políticas. Sevilla, Universidad Pablo Olavide/AGE.

Fernández, G. y Quirós, F. (2010): Atlas Temático de España, vols. II y IV. Oviedo, Ediciones Nobel.

García, M. y Delgado Orusco, E. (2010): Guía de Integración Paisajística de los Polígonos Industriales y Parques Empresariales de Cantabria. Santander, SICAM.

Gil Álvareaz, E. (2008): "Estrategias de promoción y ordenación de la industria en San Sebastián y su área de influencia". Lurralde, 31, pp. 168-188.

Gómez Delgado, M. y Barredo Cano, J. I. (2005): Sistemas de Información Geográfica y Evaluación Multicriterio en la ordenación del territorio. Madrid, Editorial Ra-Ma.

Humbert, A.; Molinero, F. y Valenzuela, M. (coords.) (2011): España en la Unión Europea. Un cuarto de siglo de mutaciones territoriales. Madrid, Casa Velázquez. 
Lázaro, J. A.; Sendín, M. A. (2002): "La producción de suelo industrial a través del ejemplo de Gijón”, en: Actas III Congreso Internacional de Ordenación del Territorio. Madrid, Fundicot, pp.745-761.

López González, A. y Somoza Medina, J. (2012): "La evolución de la estrategias de suelo industrial y el papel de la Administración Pública en España. El plan de suelo empresarial de Galicia”, en: Royé, D. et al.: Actas XIII Coloquio Ibérico de Geografía. Santiago de Compostela, Meubook (s.p.).

Martínez, L. C. y Luengo, J. A. (2005): "Localización de la actividad manufacturera en Castilla y León. Los dinamismos espaciales inducidos por la industria". Ería. Revista de Geografía, 67, pp. 155-172.

Méndez, R. (1997): Geografía Económica. La lógica espacial del capitalismo global. Barcelona, Ariel Geografía.

Méndez, R. (ed.) (2010): Estrategias de innovación industrial y desarrollo económico en las ciudades intermedias de España. Madrid, Fundación BBVA.

Molina, I. (2005): "La influencia de la accesibilidad en las estrategias públicas de creación de suelo industrial. El ejemplo de las campiñas meridionales de Castilla y león”, en Actas XIX Congreso de Geógrafos Españoles. Santander, AGE y Departamento de Geografía y OT de la Universidad de Cantabria (CD).

Nogués Linares, S. et al. (2007): "El suelo y las nuevas formas del espacio industrial", en Martínez, F. J. (coord.): Cantabria 2020. El horizonte de la industria. Santander, CEOE/CEPYME, pp. 122-131.

Ojeda Zujar, J.; Vallejo Villalta, I. y Pita Lopez, M. F. (2010): "La información geográfica al servicio de los ciudadanos: de lo global a lo local", en: XVI Congreso Nacional de Tecnologías de la Información Geográfica. Sevilla, Secretariado de Publicaciones de la Universidad de Sevilla (CD).

Instituto Geográfico Nacional (2008): ORDEN FOM/956/2008, de 31 de marzo, por la que se aprueba la política de difusión pública de la información generada por la Dirección General del Instituto Geográfico Nacional, BOE del 8 de abril de 2008.

Peters, D. (2008): Building a GIS: System ArchitectureDesing Strategies for managers. Redlands, USA, Editorial Esri-Press.

Pujadas, R. y Font, J. (1998): Ordenación y planificación territorial. Madrid, Síntesis.

Rodríguez González, R. (1999): "Política de oferta de suelo empresarial en Galicia", en: Actas VI Jornadas de Geografía Industrial, pp. 265-276. Alicante, Universidad de Alicante.

Romero, J. y Farinós, J. (eds.) (2004): Ordenación del territorio y desarrollo territorial. El gobierno del territorio en Europa: tradiciones, contextos, culturas y nuevas visiones. Gijón, Ediciones Trea.

Ruiz Urrestarazu, E. y Galdós Urrutia, R. (2002): "Continuidad de la industria: estructura y localización industrial en el País Vasco". Ería. Revista de Geografía, 59, pp. 357-370.

Tomlinson, R. (2008): Pensando en el SIG. Redlands-USA,Editorial Esri-Press. 
Torres Enjuto, C. (1995): Industria y Territorio en Bizkaia. Vitoria, Instituto Vasco de Administración Pública. Colección Tesis Doctorales.

Vries, A. De y Sesma Goñi, A. (2009): "Criterios de ordenación territorial en la planificación de polígonos industriales: el caso de Navarra", en: Actas XXXV Reunión de Estudios Regionales. Valencia, Asociación Española de Ciencia Regional, pp. 1-24

Wilson, A. G. (1980): Geografía y planeamiento urbano y regional. Barcelona, OikosTau.

\section{FUENTES ELECTRÓNICAS}

ADE Parques Tecnológicos y Empresariales. [En línea]. Castilla y León, Junta de Castilla y León. http://www.adeparques.es/. (Fecha de verificación: 22/03/2015).

FUNDACIÓN ADEUROPA E INVERTIR EN CASTILLA Y LEÓN. [En línea]. Suelos Industriales. Castilla y León, Fundación Adeuropa. http://www.invertirencastillayleon.com/svpeSueloExtMapaCn.do?method=buscarMapa (Fecha de verificación: 22/03/2015).

Grupo Sodercan. Buscador de suelo. [En línea]. Santander, http://www.sican.es/sican/ buscador_de_suelo/ (Fecha de verificación: 22/03/2015).

Instituto de Desarrollo Económico del Principado de Asturias. [En línea]. Localizador de espacio industrial de Asturias (LINEA). Asturias, IDEPA. http://www.idepa.es /sites/web/idepaweb/servicios/infraestructuras_industriales/sig (Fecha de verificación: 22/03/2015).

Ministerio de Fomento. Sociedad Estatal de Promoción y Equipamiento del Suelo [En línea]. España, SEPES. http://www.sepes.es/ (Fecha de verificación: 22/03/2015).

Navarra de Suelo Industrial, SA. [En línea]. Pamplona, NASUINSA. http://www.nasuinsa.es/ (Fecha de verificación: 22/03/2015).

Sociedad para la Promoción de Suelo y Construcciones Industriales, SA. [En línea]. Bilbao, SPRILUR. http://www.sprilur.es/grupo-sprilur/ (Fecha de verificación: 22/03/2015).

\section{RESUMEN}

Trabajo centrado en aspectos metodológicos ligados al estudio geográfico de las áreas empresariales en España. Se trata de exponer, en primer lugar, el proceso de captación y sistematización de datos, tanto cuantitativos como cualitativos, para el análisis a escala regional y local de las políticas de ordenación y planificación de las áreas empresariales en un ámbito que integra a la mayoría de las Comunidades Autónomas del norte del país y con un enfoque que contempla la diversidad de polígonos y parques empresariales promovidos por diferentes agentes públicos. El segundo objetivo es explicar las técnicas aplicadas para representar la información significativa sobre distri- 
bución y localización de las áreas empresariales y obtener una herramienta que permita la comprensión del actual modelo territorial de la industria.

PAlabras Clave: Metodología sobre áreas empresariales; tipología polígonos industriales; sistemas de información geográfica; norte de España.

\begin{abstract}
Work focused on methodological issues related to studying geographic business areas in Spain. It is present in the first place, the information gathering process and both quantitative and qualitative data for analysis at regional and municipal industrial areas consolidated in an area that covers most of the northern regions of the country and to explain, secondly, the techniques used to interpret and represent the relevant information on the distribution and location of productive spaces in that territory
\end{abstract}

KEY WORDS: Methodology on business are; typology of industrial estates as; GIS; northern Spain.

\title{
RÉSUMÉ
}

Travaux ont porté sur des aspects méthodologiques liés à l'étude géographique des quartiers d'affaires en Espagne. Il est, tout d'abord, d'exposer le processus de collecte et systématisation des données, tant quantitatives que qualitatives, pour analyse, aux niveaux régional et local, des politiques de gestion et de planification des quartiers d'affaires dans un domaine qui intègre la plupart des communautés autonomes du Nord du pays et avec une approche qui tienne compte de la diversité des polygones et des parcs d'activités promues par divers responsables gouvernementaux. Le second objectif est d'expliquer les techniques appliquées représentent d'importantes informations sur la distribution et l'emplacement des quartiers d'affaires et d'obtenir un outil qui permet la compréhension et l'explication du modèle territorial actuel de l'industrie.

MotS CLÉs: Méthodologie sur zonas pour les entreprises; typologie zones industrielles; systèmes d'information géographique; Nord de Espana. 